\title{
Lifestyle intervention by group-based rehabilitation versus individual counseling in type 2 diabetes: 1 -year follow-up of the Copenhagen type 2 diabetes rehabilitation project
}

\author{
Eva Soelberg Vadstrup ${ }^{1^{*}}$, A. Frølich ${ }^{2}$, H. Perrild ${ }^{1}$, E. Borg ${ }^{3}$, M. Røder ${ }^{4}$ \\ ${ }^{1}$ Department of Endocrinology and Gastroenterology, Bispebjerg University Hospital, Copenhagen, Denmark; \\ *Corresponding Author: eva.vadstrup@gmail.com \\ ${ }^{2}$ Department of Integrated Healthcare, Bispebjerg University Hospital, Copenhagen, Denmark \\ ${ }^{3}$ Health Care Center Oesterbro, Copenhagen, Denmark \\ ${ }^{4}$ Department of Cardiology and Endocrinology, Hillerød University Hospital, Hillerød, Denmark
}

Received 8 June 2012; revised 9 July 2012; accepted 20 July 2012

\begin{abstract}
Background: Today most guidelines on the management of type 2 diabetes incorporate lifestyle intervention including patient education, physical activity and dietary modification. However, the content and organization of lifestyle intervention programs are still debatable. Aims: To compare the longer term effectiveness of a group-based rehabilitation program with an individual counseling program at improving glycemic control, cardiovascular risk factors and quality of life among type 2 diabetes patients.

$1.6 \mathrm{~cm}(95 \% \mathrm{Cl}=-2.5,-0.6)$. Vitality, fatigue distress, physical functioning and cardiovascular distress improved over time $(P<0.05)$ in the two groups combined. Repeated measurement analysis did not result in significant differences between the groups of any outcome. Conclusions: This study demonstrates that group-based rehabilitation in a primary healthcare center is a comparable alternative to individual counseling in an outpatient clinic. However, the resource use of the rehabilitation program was twice as much as the individual program.
\end{abstract} Methods: We randomized 143 type 2 diabetes patients to either a 6-month group-based rehabilitation program, including patient education, supervised exercise, and diet intervention, or a 6-month individual counseling program. Followup time was 12 months after baseline. Outcome measures were glycated hemoglobin $\left(\mathrm{HbA}_{1 \mathrm{c}}\right)$, cardiovascular risk factors, quality-of-life and self-rated health. Results: In the rehabilitation group, $\mathrm{HbA}_{1 \mathrm{c}}$ decreased $0.2 \%$-point $(95 \%$ confidence interval $[\mathrm{Cl}]=-0.4,-0.03$ ), systolic blood pressure decreased $6 \mathrm{mmHg}(95 \% \mathrm{Cl}=-9.3$, -2.5), diastolic blood pressure decreased 4 $\mathrm{mmHg}(95 \% \mathrm{Cl}=-6.3,-2.4)$, weight decreased $2.2 \mathrm{~kg}(95 \% \mathrm{Cl}=-3.2,-1.3)$, and waist circumference decreased $2.0 \mathrm{~cm}(95 \% \mathrm{Cl}=-2.9,-1.1)$. In the individual group, $\mathrm{HbA}_{1 \mathrm{c}}$ decreased $\mathbf{0 . 4 \%}$ $(95 \% \mathrm{Cl}=-0.6,-0.1)$, systolic blood pressure decreased $3 \mathrm{mmHg}(95 \% \mathrm{Cl}=-6.3,-0.7)$, diastolic blood pressure decreased $3 \mathrm{mmHg}(95 \%$ $\mathrm{Cl}=-4.7,-0.7)$, weight decreased $1.6 \mathrm{~kg}(95 \% \mathrm{Cl}$ $=-2.6,-0.7)$, and waist circumference decreased
Keywords: Rehabilitation; Lifestyle Intervention; Glycemic Control; Quality of Life; Cardiovascular Risk Factors

\section{INTRODUCTION}

Lifestyle intervention plays an important role in the management of type 2 diabetes. Educational interventions with or without physical activity and dietary advice have resulted in beneficial effects on glycemic control [1-3], diabetes knowledge [4,5] and quality of life [6]. However, the content, duration, setting, and ways of delivering diabetes education are still controversial. Since the early 1990 there has been an increasing focus on patient-centered perspectives, goal setting, self-management and empowerment [7]. Lack of time and resources have encouraged clinics to offer patients group-based education programs rather than individual one-to-one education. Compared with individual education, groupbased education were equally or more effective at improving glycated hemoglobin $\left(\mathrm{HbA}_{1 \mathrm{c}}\right)$, cardiovascular risk factors and quality of life $[8,9]$. Modification of life- 
style behaviors in the long term, however, appears to be difficult. Many type 2 diabetes patients engage in new lifestyle behaviors for short periods, but are usually unable to maintain these changes over time [5].

A primary healthcare center in Eastern Copenhagen was established for provision of a group-based rehabilitation program to patients with chronic diseases, including type 2 diabetes. The Copenhagen Type 2 Diabetes Rehabilitation Project was designed to evaluate the effectiveness of a 6-month group-based rehabilitation program compared with individual counseling in type 2 diabetes patients [10]. Rehabilitation is a goal-orientated and time-limited collaboration process between the patients and the multi-disciplinary team providing patients with self-management skills to support change in lifestyle. We hypothesized that a group-based rehabilitation program would improve glycemic control, cardiovascular risk factors and quality of life in type 2 diabetes patients more than an individual counseling program. The short-term effects of the 6-month intervention-period were previously published $[11,12]$ and resulted in a significantly larger decrease in $\mathrm{HbA}_{1 \mathrm{c}}$ in the individual group. Most cardiovascular risk factors, vitality and fatigue distress improved equally in both groups. This paper reports the longer term follow-up effects 12 months after baseline.

\section{PATIENTS AND METHODS}

\subsection{Study Design and Subjects}

The study deign was described in detail previously [10-12]. Briefly, we screened 264 type 2 diabetes patients of which 143 met the following inclusion criteria: Type 2 diabetes patients (age $\geq 18$ years) with $\mathrm{HbA}_{\mathrm{cc}} \geq 6.8 \%$ and $\leq 10.0 \%$. All patients gave their informed consent to the study, which conformed to the principles of the Declaration of Helsinki. Patients were stratified by gender and age. Using consecutively numbered sealed envelopes patients were randomly assigned to a 6-month groupbased rehabilitation program in a primary healthcare center or to a 6-month individual counseling program in a diabetes outpatient clinic. Neither the investigator nor the patients were blinded to the allocation. The study was approved by the local ethnics committee. Clinical Trials. gov registration number: NCT00284609.

\subsection{Interventions}

The group-based rehabilitation program at the healthcare center was derived from evidence-based guidelines and from the empowerment model and involved a great amount of patient collaboration $[13,14]$. The program focused on achievement of self-selected goals and enhanced control and problem solving strategies.

All groups comprised of 8 patients. The education component consisted of 90-min sessions held weekly for a total of 6 weeks. The curriculum included: pathology of diabetes, self blood-glucose monitoring, physical activity and change in diet as a therapy, the diabetic foot, and diabetic complications. The education team consisted of a nurse, a dietician, a physiotherapist, and a podiatrist. All personnel were trained and supervised in the use of the motivational interviewing technique by an expert psychologist [15].

Patients participated in supervised exercise in 1.5 -hour sessions twice a week in a 12-week period. A physiotherapist tailored an individual program for each patient including both aerobic and resistance exercise. The diet instruction classes were taught by a dietician and included two cooking classes, each of three hours, and a 2-hour session in a local supermarket.

Before patients entered the program they participated in a motivational interview and set personal goals [15]. Goal achievement was evaluated in collaboration with the patients at the end of the program and at 1 and 3 months after program completion by telephone contacts.

The individual counseling program at the diabetes outpatient clinic at Bispebjerg University Hospital was based on the same clinical guidelines and empowerment approach $[13,14]$. The nurse specialist and the dietician were trained in the use of the motivational interviewing technique [15].

During a period of 6 months patients were scheduled for 4 one-hour sessions of individual counseling with a diabetes nurse specialist. Using the patients' own stories they received personalized information and guidance about type 2 diabetes, medications, risk factors, bloodglucose self-monitoring, and increasing level of physical activity to the recommended 30 minutes of daily exercise.

The program also included 3 individual counseling sessions with a dietician. The patients' set personal goals and the dietician developed in cooperation with the patient a diet-schedule based on biochemical, anthropometrical and medical records and patients' motivation and attitudes. In addition, the patients received 45 minutes guidance and instructions about foot-care from a podiatrist.

The endocrinologist or general practitioner caring for the patients prior to the study continued to provide diabetes management during and after the intervention; however, they were not part of the study team. Patients reported any medical change to the study team; these were quantified by the principal investigator and included in the analysis.

\subsection{Outcomes}

The primary outcome measure was change in glycemic control, as indicated by $\mathrm{HbA}_{1 \mathrm{c}}$ levels. Other pre- 
defined outcomes included blood pressure, lipid profile, body weight, waist circumference, health-related quality of life (HRQOL), self-rated health, insulin resistance and beta-cell function estimates.

\subsection{Measurements}

Demographic and clinical parameters were recorded at baseline and repeated at the completion of the 6-month intervention program and 12 months after baseline. A detailed description of blood sample measurements and clinical parameters were previously published $[10,11]$.

The SF-36 questionnaire (Medical Outcomes Study Short Form 36-item Health Survey, version 1.0) was used to measure health-related quality of life in both physical and mental health component scores [16]. Eight subscales were generated in the following domains: physical functioning, physical limitation, bodily pain, general health, vitality, social functioning, emotional limitation, and mental health. An increased score (0 - 100) on SF-36 indicated an improvement.

Self-rated diabetes symptoms were measured using the DSC-R questionnaire (Diabetes Symptom ChecklistRevised) [17]. The DSC-R measures the perceived burden (from 0 - 5 points) of diabetes-related symptoms. The 34 items were grouped into 8 subscales: hyperglycemia, hypoglycemia, psychological cognitive functioning, psychological fatigue, cardiovascular symptoms, neuropathic pain, neuropathic sensory, and ophthalmologic functioning. A decreased score on DSC-R indicated an improvement. The validity and reliability of the SF-36 and the DSC-R instrument has been well documented $[18,19]$.

\subsection{Statistical Analyses}

We calculated the sample size of 160 patients in order to yield $90 \%$ power ( $\alpha=0.05$, two-tailed) to detect an absolute difference in $\mathrm{HbA}_{1 \mathrm{c}}$ of $0.7 \%$-point between the two groups. The study statistician carrying out the data analysis was blinded to patients' allocation.

The change over time (repeated measurement) was analyzed using mixed models (PROC MIXED) in SAS 9.2. (Cary, NC) [20]. The linear mixed model assumptions appeared satisfactory since model tests showed that the residuals of the majority of variables were almost normal distributed. Logarithmic transformation was not possible because some of the variables had the value "zero". The setup included a random intercept as all measurements from the same person were correlated with the same correlation coefficient and all missing observations were considered to be missing at random. All available data were used in the analysis. However, due to high dropout rate an additional intention-to-treat (ITT) analysis was done (for glycemic control and cardiovas- cular risk factors) with imputed values for missing data by carrying forward the last measured outcome. ITT analysis was not possible for quality-of-life and selfrated health measurements since the majority of missing data included baseline data.

Group $\times$ time interactions represent differences in the shape of the response profile over time (baseline, 6 months and 12 months) by group. Time represents the main effects of time from baseline to 12 months follow-up in the two groups combined. We used two-sided tests and a statistical significance level at $P<0.05$.

\section{RESULT}

Baseline characteristics of the study population are shown in Table 1. Of the 143 randomized patients 120 completed the 12 months visit. Main reasons for dropout were time constraints, disappointment with the allocation, and personal reasons. There were no difference in baseline values between dropouts and patients who continued follow-up with the single exception that dropouts had higher weight and waist circumference.

Adherence to the intervention programs was judged by session attendance. In the rehabilitation group, 37 (64\%) patients attended at least 18 of 24 exercise sessions, 42 (72\%) patients at least five of six education sessions, and $50(86 \%)$ patients attended at least two of three dietary education sessions. In the individual group, 48 (84\%) patients attended at least three of four nurses counseling

Table 1. Baseline characteristics of patients in the rehabilitation group and in the individual group.

\begin{tabular}{ccc}
\hline & $\begin{array}{c}\text { Rehabilitation } \\
\text { group }\end{array}$ & $\begin{array}{c}\text { Individual } \\
\text { group }\end{array}$ \\
\cline { 2 - 3 } & $N=70$ & $N=73$ \\
\hline Age & $58.5(9.0)$ & $58.0(10.3)$ \\
Men, n (\%) & $41(59)$ & $44(60)$ \\
Duration of T2DM, years (range) & $6.7(0-37)$ & $6.4(0-24)$ \\
HbA Ic $_{0}$ & $7.9(0.8)$ & $7.8(0.9)$ \\
Blood pressure, mmHg & $146(18)$ & $145(17)$ \\
Systolic & $85(10)$ & $84(9)$ \\
Diastolic & $96.2(15.2)$ & $98.2(24.8)$ \\
Weight, kg & $108.9(12.8)$ & $108.6(16.9)$ \\
Waist circumference, cm & $4.7(1.0)$ & $4.9(1.1)$ \\
Total cholesterol & $1.2(0.3)$ & $1.2(0.4)$ \\
HDL-C & $2.7(0.9)$ & $2.6(1.0)$ \\
LDL-C & $2.2(1.4)$ & $2.4(1.6)$ \\
Triglycerides & &
\end{tabular}


sessions, and $50(88 \%)$ patients attended at least two of three dietician counseling sessions.

\subsection{Glycemic Control}

Figure 1 shows the change over time in $\mathrm{HbA}_{1 \mathrm{c}}$ from baseline to 12 months follow-up. Change of $\mathrm{HbA}_{1 \mathrm{c}}$ did not differ between the two groups $(P=0.23)$. $\mathrm{HbA}_{1 \mathrm{c}}$ decreased by $0.2 \%$-point $(95 \%$ confidence interval $[\mathrm{CI}]=$ -0.4 to 0.03 ) in the rehabilitation group and by $0.4 \%$ point $(95 \% \mathrm{CI}=-0.6$ to -0.1$)$ in the individual group compared with baseline values. There was a significant effect of time in the two groups combined $(P<0.001)$. The intention-to-treat (ITT) analysis did not change the results.

The change over time in HOMA-IR, HOMA-B and proinsulin/insulin ratio did not differ between the two groups (Table 2). The ITT analysis of HOMA-IR, HOMA-B and proinsulin/insulin ratio did not change the results.

\subsection{Cardiovascular Risk Factors}

The change over time in weight, waist circumference and blood pressure did not differ significantly between the two groups (Figure 2). The systolic blood pressure decreased by $6 \mathrm{mmHg}(95 \% \mathrm{CI}=-9.3$ to -2.5$)$ in the rehabilitation group but only by $3 \mathrm{mmHg}(95 \% \mathrm{CI}=-6.3$ to 0.7 ) in the individual group. The diastolic blood pressure decreased by $4 \mathrm{mmHg}(95 \% \mathrm{CI}=-6.3$ to -2.4$)$ in the rehabilitation group and by $3 \mathrm{mmHg}(95 \% \mathrm{CI}=-4.7$ to -0.7$)$ in the individual group. Body weight was reduced by $2.2 \mathrm{~kg}(95 \% \mathrm{CI}=-3.2$ to -1.3$)$ in the rehabilitation group and by $1.6 \mathrm{~kg}(95 \% \mathrm{CI}=-2.6$ to -0.7$)$ in the individual group. Waist circumference decreased by $2 \mathrm{~cm}$
$(95 \% \mathrm{CI}=-2.9$ to -1.1$)$ in the rehabilitation group and by $1.6 \mathrm{~cm}(95 \% \mathrm{CI}=-2.5$ to -0.6$)$ in the individual group. There was a significant effect of time in all these outcomes in the two groups combined $(P<0.001)$. The changes of plasma lipids between and within the two groups over time were very small and statistically insignificant (data not shown).

The ITT analysis did not change the results.

\subsection{SF-36}

There were no significant differences in change over time in any of the 8 subscales between the rehabilitation group and the individual group. Table 2 shows the results of 4 subscales in which significant changes within the groups occurred. There was a significant improvement in physical function in the rehabilitation group (4.4

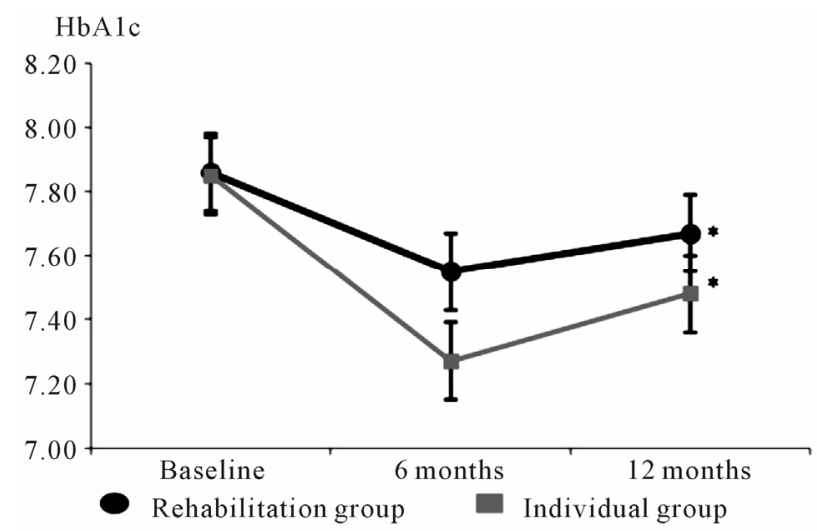

Figure 1. Mean values of $\mathrm{HbA}_{1 \mathrm{c}}$ at baseline, 6 months and 12 months follow-up in the rehabilitation group and in the individual group. Time: $P<0.001$ Group $\times$ time: $P=0.21^{*} P<$ 0.05 within group ( 12 months compared with baseline values).

Table 2. Change in HOMA-IR, HOMA-B and proinsulin/insulin ratio from baseline to 12 months.

\begin{tabular}{lcccc}
\hline & Rehabilitation group & Individual group & \multicolumn{2}{c}{$P$} \\
\cline { 2 - 5 } & Mean (SEM) & Mean (SEM) & Time & Group $\times$ time \\
\hline HOMA-IR $^{*}$ & $3.8(0.4)$ & $4.5(0.3)$ & & \\
Baseline & $3.5(0.4)$ & $3.8(0.4)^{\dagger}$ & $<0.001$ & 0.60 \\
12 months & & & & \\
HOMA-B & & & \\
Baseline & $41.0(4.8)$ & $40.5(4.5)$ & 0.24 \\
12 months & $48.9(5.0)$ & $47.2(4.9)$ & & \\
Proinsulin/insulin ratio & & & & \\
Baseline & $37.3(1.7)$ & $35.0(1.6)$ & 0.06 & 0.69 \\
12 months & $34.9(1.7)$ & $34.2(1.7)$ & 0.06 & \\
\hline
\end{tabular}

The repeated measurement analysis of change over time from baseline to 12 months follow-up. * Only in patients not in insulin treatment. HOMA-IR = Homeostasis Model Assessment of Insulin Resistance, HOMA-B = Homeostasis Model Assessment of beta-cell function. ${ }^{\dagger} P<0.05$ within group (12 months compared with baseline values). 

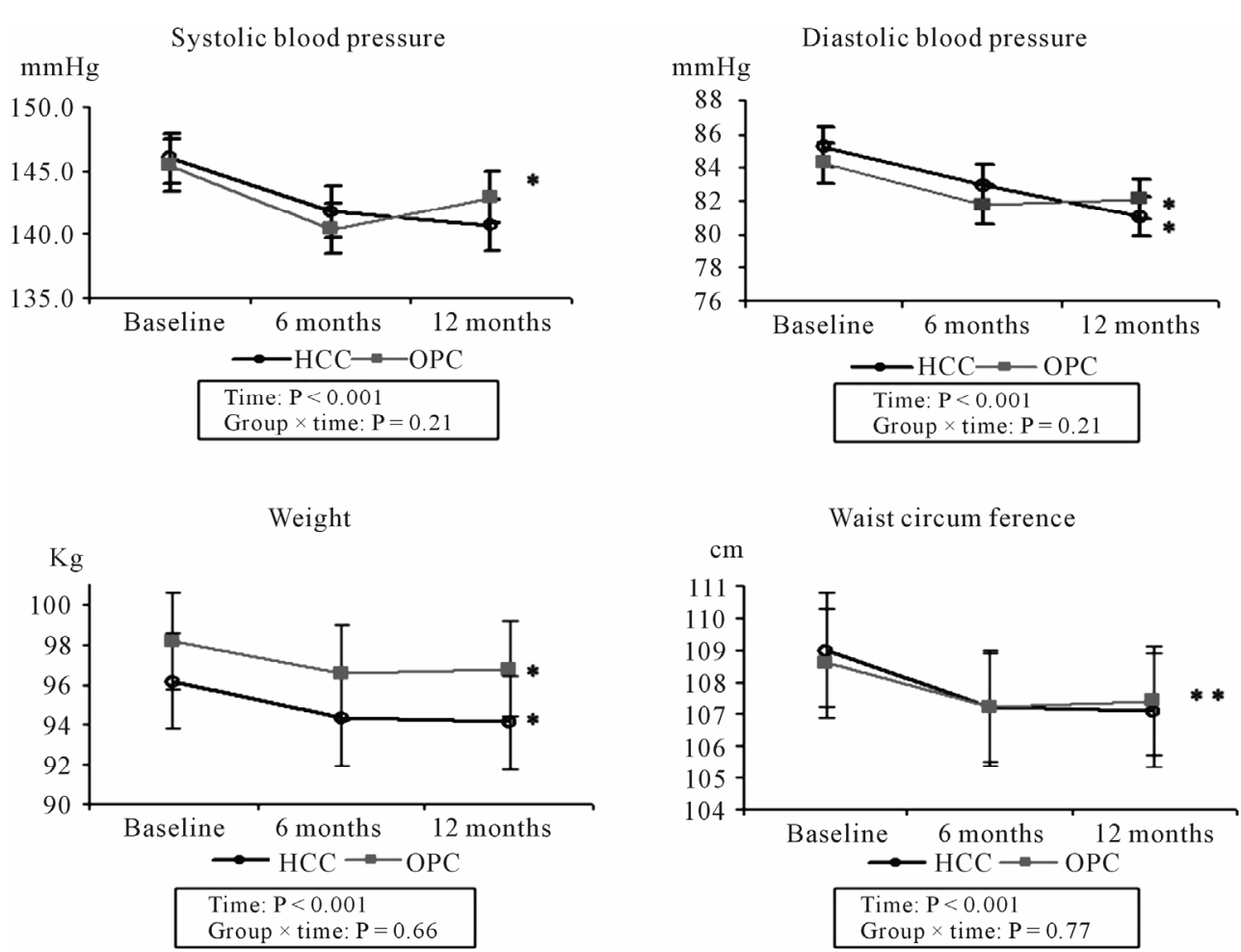

Figure 2. Mean values of blood pressure, weight and waist circumference at baseline, 6 months and 12 months follow-up. HCC $=$ Healthcare center (rehabilitation group). OPC $=$ Outpatient clinic (individual group). ${ }^{*} P<0.05$ within group (12 months compared with baseline values).

point, $P=0.03$ ). General health had improved from baseline values in the individual group (4.8 point, $P=0.03$ ) and furthermore there was a significant difference between the two groups in mental health in favor of the individual group ( 7.5 point, $P=0.02$ ). There was a significant effect of time in vitality and physical functioning in the two groups combined $(P<0.001)$.

\subsection{DSC-R}

There were no significant differences in change over time in hyper- and hypoglycemic distress or any of the other DSC-R subscales between the two groups (Table 3). There was a significant decrease in fatigue distress $(-7.2$ point, $P=0.003)$ and cardiovascular distress $(-3.7$ point, $P=0.03)$ in the rehabilitation group compared with baseline values. In the two groups combined there was a significant effect of time in fatigue and cardiovascular distress $(P<0.05)$.

\section{DISCUSSION}

From baseline to 12 months follow-up, we found no significant differences over time between 6-month groupbased rehabilitation and 6-month individual counseling on glycemic control, cardiovascular risk factors, HRQOL, or self-rated health in patients with type 2 diabetes.
The change in $\mathrm{HbA}_{1 \mathrm{c}}$ in the rehabilitation group was less than expected from other educational and exercise studies $[1,21]$. In the individual group, the decrease in $\mathrm{HbA}_{1 \mathrm{c}}$ was still significant after 12 months compared with baseline but not in the rehabilitation group. In both groups $\mathrm{HbA}_{1 \mathrm{c}}$ tended to increase again. These results confirm what was found in a meta-analysis by Norris et al that the longer the follow-up after an educational intervention, the lesser the effect on $\mathrm{HbA}_{1 \mathrm{c}}$ [2]. In most studies the maximal effect on $\mathrm{HbA}_{1 \mathrm{c}}$ was no longer than 6 months.

In the same meta-analysis, $\mathrm{HbA}_{1 \mathrm{c}}$ decreased more with additional contact time between patient and educators. This was not the case in our study since the patients in the rehabilitation program had 57.5 hours of contact whereas the patients in the individual group had 6.75 hours of contact. In addition, the personnel resource use in the rehabilitation program was twice as much as in the individual program (13.65 personnel hours versus 6.75 personnel hours).

At the 12-month follow-up visit, the mean diastolic blood pressure decreased by $4 \mathrm{mmHg}$ and the mean systolic blood pressure decreased by $6 \mathrm{mmHg}$ in the rehabilitation group compared with baseline values. If sustained, these results are clinically important. Epidemiological studies have shown that a long-term difference of $5-6 \mathrm{mmHg}$ in diastolic blood pressure was associated 
Table 3. Change in health-related quality-of-life (SF36) and self-rated health (DSC-R) from baseline to 12 months.

\begin{tabular}{lcccc}
\hline & Rehabilitation group & Individual group & \multicolumn{2}{c}{$P$} \\
\cline { 2 - 5 } & Mean (SEM) & Mean (SEM) & Time & Group $\times$ time \\
\hline SF36 $^{*}$ & & & &
\end{tabular}

Physical function

$\begin{array}{lrrrr}\text { Baseline } & 77.8(2.4) & 83.0(2.4) & & \\ 12 \text { months } & 82.2(2.4) \dagger & 85.2(2.4) & <0.05 & 0.73\end{array}$

General health

$$
\text { Baseline }
$$

12 months

Vitality

Baseline

12 months

Mental health

Baseline

\section{DSC-R ${ }^{\ddagger}$}

Hyperglycaemia

Baseline

Hypoglycaemia

12 months

Fatigue

$$
\text { Baseline }
$$

Cardiology

Baseline

12 months

Total

$$
\text { Baseline }
$$

12 months

Baseline

12 months
63.1 (2.6)

$64.1(2.5)$

58.3 (2.9)

$62.2(2.9)$

75.9 (2.3)

75.4 (2.3)

$1.38(0.15)$

$1.40(0.14)$

$1.16(0.13)$

$1.05(0.12)$

$2.13(0.15)$

$1.77(0.15)^{\dagger}$

$0.96(0.10)$

$0.77(0.10)^{\dagger}$

$1.04(0.08)$

$0.99(0.08)$
64.9 (2.6)

$69.8(2.6)^{\dagger}$

0.07

0.41

$62.1(2.9)$

$66.5(3.0)$

$<0.01$

0.95

78.8 (2.3)

$82.9(2.4)^{\natural}$

0.26

0.28

$1.51(0.14)$

$1.37(0.15)$

0.45

0.28

$1.01(0.13)$

$0.95(0.13)$

0.36

0.09

$1.86(0.15)$

$1.73(0.15)$

$<0.01$

0.38

$0.71(0.10)$

$0.66(0.10)$

$<0.05$

0.15

"Score scale range $(0-100)$. A higher score on SF-36 indicates an improvement in quality of life. ${ }^{\star}$ Score scale $(0-5)$. A lower score indicate an improvement; ${ }^{\dagger} P<0.05$ within group (12 months compared with baseline values); ${ }^{{ }^{\dagger}} P<0.05$ comparison between groups at one particular time (12 months follow-up). with about $40 \%$ lower risk of stroke and about $30 \%$ lower risk of ischemic heart disease (IHD) [22,23]. Even a long-term difference of $2 \mathrm{mmHg}$ in systolic blood pressure would cause about $10 \%$ lower stroke mortality rate and about 7\% lower mortality rate from IHD [23].

In obese patients participating in short-term behavioral intervention studies the point of maximal weight loss was 6 months after the initiation of the intervention and then the weight regain began [24]. Although the shortterm reduction in weight and waist circumference in our study only accounted for $2 \%$ of the baseline values, it sustained after 12 months in both groups.

Only in the rehabilitation group, the improvements in fatigue and cardiovascular distress sustained statistically significant after 12 months. The physical function in the rehabilitation group and general health in the individual 
group also improved. We previously showed that hyperand hypoglycemic distress improved significantly in the individual group and compared with the rehabilitation group after the 6-month intervention period [12]. These differences did not sustain after 12 months. In general, the changes over time in HRQOL and self-rated health were relatively small in both groups. Compared with study populations of uncontrolled type 2 diabetes patients our study population had higher baseline scores of HRQOL and lower baseline scores of self-rated symptom distress $[25,26]$. If an individual is functioning at an almost optimal level prior to an intervention, it might be difficult to demonstrate a positive effect of the intervention because there is little room for improvements. Thus, our results do not match the short and long-term improvements found in a systematic review of well-being and HRQOL in self-management interventions [27].

The current study had some limitations. Since both interventions were multi-disciplinary it was not possible to identify the effect of each component of the interventions. We did not evaluate the compliance of the educational component (i.e. by measuring patients' knowledge) or the diet intervention (i.e. by calorie intake). We did use several physical tests to measure patients' physical fitness that showed significant improvements in muscle strength and mobility after the exercise program in the rehabilitation group (data not shown). Neither the patients nor the investigator were blinded to the allocation in this study, which may have induced bias by the investigator examining the patients after the interventions. If the investigator was prejudiced in favor of the intervention group the already limited improvements found in the intervention group would be even smaller. The dropout rate was high which could lead to attrition bias. However, the only difference between dropouts and completers was that dropouts were more obese than completers. This might have resulted in a larger observed weight loss in the individual group but probably not influenced any of the other results. The use of anti-diabetic, anti-hypertensive and lipid-lowering medication was possible confounders but there were no significant differences in medication use or change in medication during the intervention and in the following observation period between the groups (data not shown).

\section{CONCLUSION}

This study compared the effects of two lifestyle intervention programs delivered in a real-world context. Both interventions reflect the current clinical care setting and therefore the results are in line with what is possible to obtain at least in our clinical setting. Our study demonstrates that group-based rehabilitation in a primary healthcare center is a comparable alternative to individual coun- seling in an outpatient clinic. However, the rehabilitation program demanded more resources and probably costs and should be improved and modified in order to result in larger and sustainable improvements. In addition, our results stress the limited value of short-term educational programs in diabetes care. Further research is needed to optimize lifestyle intervention in type 2 diabetes patients and to show whether regular reinforcement can achieve sustainable improvements.

\section{ACKNOWLEDGEMENTS}

The study was supported by grants from the Jascha Foundation, the Research Foundation of Bispebjerg Hospital, the Copenhagen Capital Region Research Foundation, the National Board of Health, the Ministry of Health and Prevention, GlaxoSmithKline, Servier Denmark, Department of Endocrinology at Bispebjerg University Hospital.

We thank laboratory technician Liselotte Spuur for laboratory assistance and statistician Anne H. Andreasen for the blinded analysis. We thank the staff at the healthcare center and the diabetes outpatient clinic for participating in the study.

\section{REFERENCES}

[1] Deakin, T., McShane, C.E., Cade, J.E. and Williams, R.D. (2005) Group based training for self-management strategies in people with type 2 diabetes mellitus. Cochrane Database of Systematic Reviews, 18, Article ID: CD003417.

[2] Norris, S.L., Lau, J., Smith, S.J., Schmid, C.H. and Engelgau, M.M. (2002) Self-management education for adults with type 2 diabetes: A meta-analysis of the effect on glycemic control. Diabetes Care, 25, 1159-1171. doi:10.2337/diacare.25.7.1159

[3] Gary, T.L., Genkinger, J.M., Guallar, E., Peyrot, M. and Brancati, F.L. (2003) Meta-analysis of randomized educational and behavioral interventions in type 2 diabetes. The Diabetes Educator, 29, 488-501. doi: $10.1177 / 014572170302900313$

[4] Brown, S.A. (1990) Studies of educational interventions and outcomes in diabetic adults: A meta-analysis revisited. Patient Education and Counseling, 16, 189-215. doi:10.1016/0738-3991(90)90070-2

[5] Norris, S.L., Engelgau, M.M. and Narayan, K.M. (2001) Effectiveness of self-management training in type 2 diabetes: A systematic review of randomized controlled trials. Diabetes Care, 24, 561-587. doi:10.2337/diacare.24.3.561

[6] Zhang, X., Norris, S.L., Chowdhury, F.M., Gregg, E.W. and Zhang, P. (2007) The effects of interventions on health-related quality of life among persons with diabetes: A systematic review. Medical Care, 45, 820-834. doi:10.1097/MLR.0b013e3180618b55

[7] Corabian, P. and Harstall, C. (2001) Patient diabetes education in the management of adult type-2 diabetes. Alberta Heritage Foundation for Medical Research, Edmonton.

[8] Trento, M., Passera, P., Bajardi, M., Tomalino, M., Grassi, 
G., Borgo, E., et al. (2002) Lifestyle intervention by group care prevents deterioration of Type II diabetes: A 4-year randomized controlled clinical trial. Diabetologia, 45, 1231-1239. doi:10.1007/s00125-002-0904-8

[9] Rickheim, P.L., Weaver, T.W., Flader, J.L. and Kendall, D.M. (2002) Assessment of group versus individual diabetes education: A randomized study. Diabetes Care, 25, 269-274. doi:10.2337/diacare.25.2.269

[10] Vadstrup, E.S., Frolich, A., Perrild, H., Borg, E. and Roder, M. (2009) Lifestyle intervention for type 2 diabetes patients: Trial protocol of The Copenhagen Type 2 Diabetes Rehabilitation Project. BMC Public Health, 9, 166. doi:10.1186/1471-2458-9-166

[11] Vadstrup, E.S., Frølich, A., Perrild, H., Borg, E. and Røder, M. (2011) Effect of a group-based rehabilitation programme on glycaemic control and cardiovascular risk factors in type 2 diabetes patients: The Copenhagen Type 2 Diabetes Rehabilitation Project. Patient Education and Counseling, 84, 185-190. doi:10.1016/j.pec.2010.06.031

[12] Vadstrup, E.S., Frølich, A., Perrild, H., Borg, E. and Røder, M. (2011) Health-related quality of life and self-related health in patients with type 2 diabetes: Effects of group-based rehabilitation versus individual counselling. Health and Quality of Life Outcomes, 9, 110. doi:10.1186/1477-7525-9-110

[13] Røjen, D., Vibe-Petersen, J. and Perrild, H. (2005) Education of patients with type 2 diabetes. Handbook for healthcare providers. Novo Nordisk A/S, Bagsvaerd.

[14] Funnell, M.M., Anderson, R.M., Arnold, M.S., Barr, P.A., Donnelly, M., Johnson, P.D., et al. (1991) Empowerment: an idea whose time has come in diabetes education. The Diabetes Educator, 17, 37-41. doi: $10.1177 / 014572179101700108$

[15] Miller, W.R. and Rollnick, S. (2002) Motivational interviewing: Preparing people for change. 2nd Edition, Guilford Press, New York.

[16] Ware, J.E., Jr. and Sherbourne, C.D. (1992) The MOS 36-item short-form health survey (SF-36). I. Conceptual framework and item selection. Medical Care, 30, 473-483. doi:10.1097/00005650-199206000-00002

[17] Grootenhuis, P.A., Snoek, F.J., Heine, R.J. and Bouter, L.M. (1994) Development of a type 2 diabetes symptom checklist: A measure of symptom severity. Diabetic Medicine, 11, 253-261. doi:10.1111/j.1464-5491.1994.tb00268.x

[18] McHorney, C.A., Ware, J.E., Jr., Lu, J.F. and Sherbourne,
C.D. (1994) The MOS 36-item short-form health survey (SF-36): III. Tests of data quality, scaling assumptions, and reliability across diverse patient groups. Medical Care, 32, 40-66. doi:10.1097/00005650-199401000-00004

[19] Arbuckle, R.A., Humphrey, L., Vardeva, K., Arondekar, B., Danten-Viala, M.M., Scott, J.A., et al. (2009) Psychometric evaluation of the diabetes symptom checklist-revised (DSC-R) - A measure of symptom distress. Value in Health, 12, 1168-1178.

[20] Littell, R.C., Milliken, G.A., Stroup, W.W. and Wolfinger, R.D. (1996) SAS system for mixed models. SAS Institue Inc., Cary.

[21] Thomas, D.E., Elliott, E.J. and Naughton, G.A. (2006) Exercise for type 2 diabetes mellitus. Cochrane Database of Systematic Reviews, 3, Article ID: CD002968.

[22] Collins, R., Peto, R., MacMahon, S., Hebert, P., Fiebach, N.H., Eberlein, K.A., et al. (1990) Blood pressure, stroke, and coronary heart disease. Part 2, Short-term reductions in blood pressure: Overview of randomised drug trials in their epidemiological context. Lancet, 335, 827-838. doi:10.1016/0140-6736(90)90944-Z

[23] Lewington, S., Clarke, R., Qizilbash, N., Peto, R. and Collins, R. (2002) Age-specific relevance of usual blood pressure to vascular mortality: A meta-analysis of individual data for one million adults in 61 prospective studies. Lancet, 360, 1903-1913. doi:10.1016/S0140-6736(02)11911-8

[24] Jeffery, R.W., Drewnowski, A., Epstein, L.H., Stunkard, A.J., Wilson, G.T., Wing, R.R., et al. (2000) Long-term maintenance of weight loss: Current status. Health Psychology, 19, 5-16. doi:10.1037/0278-6133.19.Suppl1.5

[25] Goddijn, P.P., Bilo, H.J., Feskens, E.J., Groeniert, K.H., van der Zee, K.I. and Meyboom-de, J.B. (1999) Longitudinal study on glycaemic control and quality of life in patients with Type 2 diabetes mellitus referred for intensified control. Diabetic Medicine, 16, 23-30. doi:10.1046/j.1464-5491.1999.00002.x

[26] Vinik, A.I. and Zhang, Q. (2007) Adding insulin glargine versus rosiglitazone: Health-related quality-of-life impact in type 2 diabetes. Diabetes Care, 30, 795-800. doi:10.2337/dc06-1712

[27] Steed, L., Cooke, D. and Newman, S. (2003) A systematic review of psychosocial outcomes following education, self-management and psychological interventions in diabetes mellitus. Patient Education and Counseling, 51, 5-15. doi:10.1016/S0738-3991(02)00213-6 